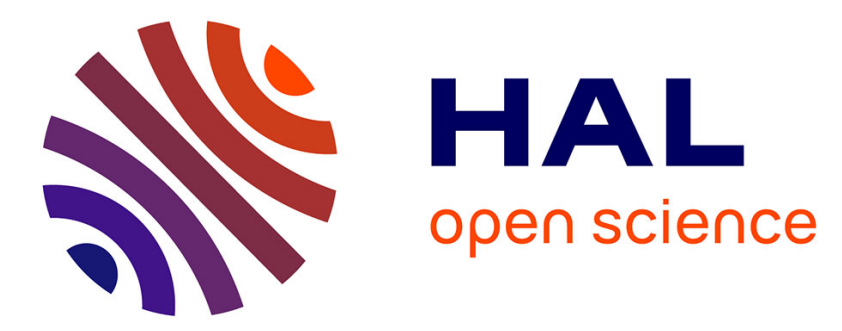

\title{
La séquence post-solutréenne du Parpallo (Espagne) : application des méthodes quantitatives de l'analyse des données à l'étude morphométrique du débitage
}

Bruno Bosselin

\section{- To cite this version:}

Bruno Bosselin. La séquence post-solutréenne du Parpallo (Espagne): application des méthodes quantitatives de l'analyse des données à l'étude morphométrique du débitage. Bulletin de la Société préhistorique française, 2001, 98 (4), pp.615-625. hal-00201610

\section{HAL Id: hal-00201610 \\ https://hal.science/hal-00201610}

Submitted on 15 Jan 2008

HAL is a multi-disciplinary open access archive for the deposit and dissemination of scientific research documents, whether they are published or not. The documents may come from teaching and research institutions in France or abroad, or from public or private research centers.
L'archive ouverte pluridisciplinaire HAL, est destinée au dépôt et à la diffusion de documents scientifiques de niveau recherche, publiés ou non, émanant des établissements d'enseignement et de recherche français ou étrangers, des laboratoires publics ou privés. 


\title{
La séquence post-solutréenne du Parpalló (Espagne) : application des méthodes quantitatives de l'analyse des données Bruno BOSSELIN à l'étude morphométrique du débitage
}

\begin{abstract}
Résumé
Une analyse quantitative de la morphométrie des produits de débitage de la séquence post-solutréenne du Parpalló est proposée. Les résultats du traitement statistique font apparaître les indices de l'existence de plusieurs systèmes de production nommés et brièvement décrits, puis leur évolution en stratigraphie est présentée. Une comparaison avec les données issues des outillages lithiques et osseux confirme la synchronisation des variations technotypologiques, malgré quelques décalages. Enfin, une conclusion rappelle les principaux apports de cette étude et propose des perspectives d'avenir.
\end{abstract}

\begin{abstract}
A quantitative analysis of the morphometry of flint debitage from the postSolutrean sequence at Parpallo is proposed. The results of the statistical treatment show indices corresponding to several production systems which are named and briefly described before their evolution in the stratigraphy is presented. Comparison with data provided by the lithic and bone tools confirms the synchronisation of the techno-typological variations, in spite of some time lags. Finally, the conclusion recalls the principal contributions of this study and proposes future perspectives.
\end{abstract}

\section{INTRODUCTION}

Depuis quelques années, l'étude technique et technologique des vestiges du débitage a permis ${ }^{1}$, en complément aux approches plus classiques de l'analyse typologique des industries lithiques et osseuses, une meilleure connaissance de l'économie des sociétés du Paléolithique supérieur. Comme le reconnaît justement A. Morala (Morala, 1993), une estimation quantitative des différentes chaînes opératoires de production présentes dans un niveau ou en stratigraphie est nécessaire pour bien comprendre les modalités, les intentions et les finalités du débitage et, corrélativement, leur influence sur la composition et le style des outillages.

Après une brève présentation historique des recherches dans la grotte du Parpalló, nous développerons une méthode d'analyse multivariée appliquée à l'étude morphométrique des supports d'outils. Les résultats du traitement statistique seront présentés, débouchant sur des indices possibles de plusieurs chaînes opératoires de débitage, dont la variabilité en stratigraphie sera analysée. Suivra alors une étude typologique des industries lithiques et osseuses, permettant de proposer une structuration de la séquence postsolutréenne du Parpalló. Finalement, une conclusion rappellera les principaux apports de cette étude, avant de poser des perspectives d'avenir.

\section{LA GROTTE DU PARPALLÓ}

Située près de Gandia, dans la province de Valencia (Espagne), la grotte du Parpalló a été découverte en 1872 par Vilanova y Piera. Elle fut fouillée par L. Pericot de 1929 à 1931. À l'entrée de la grotte, six secteurs ont été distingués : EE, Entrée Est; EO, Entrée Ouest; CE, Centre Est; CO, Centre Ouest; L, 


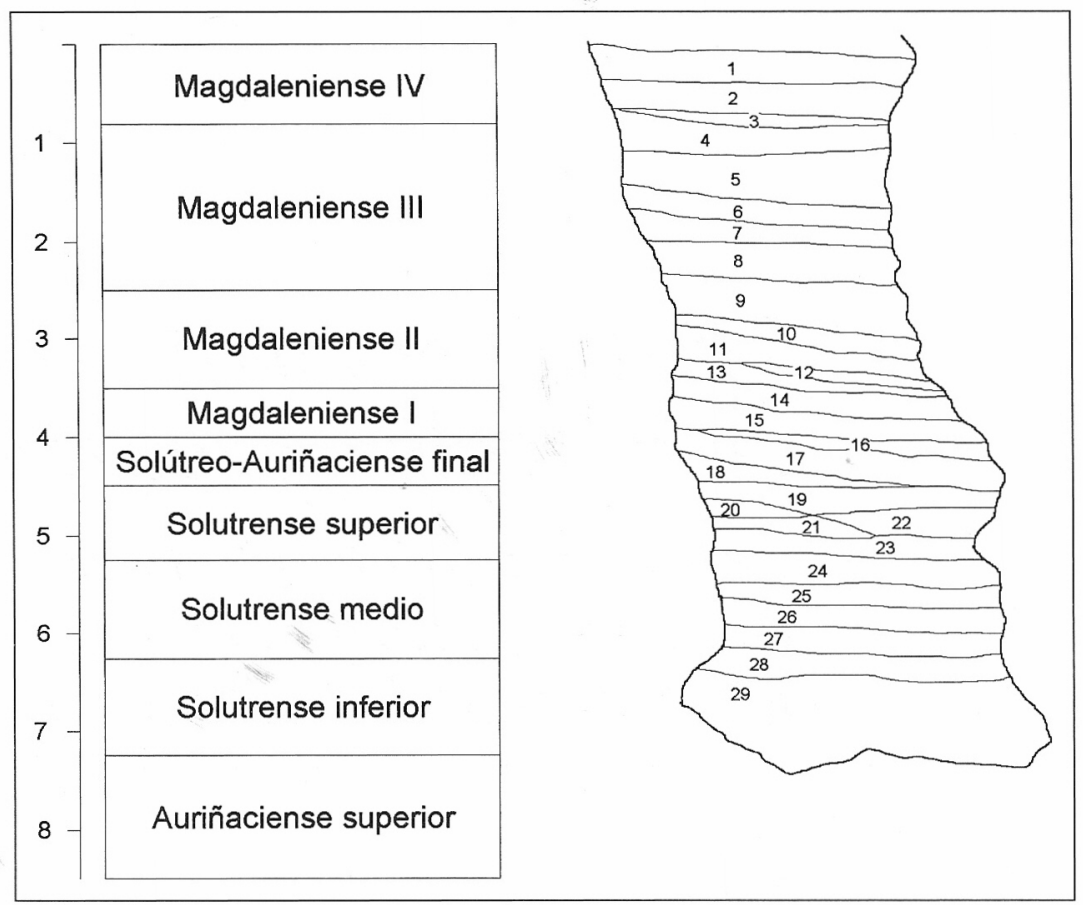

Fig. 1 - Le Parpalló : stratigraphies comparées. À gauche : séquence industrielle simplifiée de l'entrée de la grotte. À droite : coupe stratigraphique frontale du Talud.

"Levante" et P, "Ponente". Le matériel a ensuite été, à l'intérieur de ces secteurs, regroupé en trames de $25 \mathrm{~cm}$ d'épaisseur, jusqu'à une profondeur de 8,50 m. La stratigraphie est exceptionnellement longue, puisque s'étageant depuis la fin du Gravettien ("Auriñaciense superior") jusqu'au Magdalénien IV, et incluant le Solutréen, le Solutréo-Gravettien et le Magdalénien inférieur. La séquence archéologique simplifiée du Parpalló est présentée sur la figure 1a, d'après L. Pericot (Pericot, 1942).

Complémentairement, dans la partie inférieure de la grotte, un témoin a été partiellement conservé pour des recherches futures : le "Talud". Fouillé sur près de $8 \mathrm{~m}$ de profondeur en 1931 par L. Pericot (Pericot, 1942), il est constitué de 29 couches archéologiques sensiblement horizontales, d'environ 20 à $25 \mathrm{~cm}$ d'épaisseur, et différenciées selon leur coloration. La figure $1 \mathrm{~b}$ propose une représentation synthétique de la stratigraphie des niveaux reconnus dans le Talud du Parpalló.

Dans une synthèse sur les industries lithiques en Espagne, J.M. Fullola-Pericot analyse, suivant le lexique typologique de D. de Sonneville-Bordes et J. Perrot (Sonneville-Bordes et Perrot, 1954-1956), les niveaux situés entre les profondeurs de 2,5 m et 4,50 m à l'entrée de la grotte (secteurs EE, EO, CE, CO, L et P) et les couches 9 à 18 du Talud. Il propose alors une légère modification de l'articulation et de la dénomination des faciès lithiques rencontrés sur le site, sans toutefois remettre totalement en cause les conclusions de L. Pericot (Fullola-Pericot, 1979).

Pour M.J. Rodrigo-Garcia (Rodrigo-Garcia, 1988), le Solutréo-Gravettien du Parpalló se structure en deux phases typologiques et chronologiques : le SolutréoGravettien I typique à cran nombreux (ou Solutréen évolué II) dans les trames 4,75-4,50 m et 4,50-4,25 m globalisées, sans indication de provenance de secteur, et le Solutréo-Gravettien II atténué à plus rares crans (ou Solutréen évolué III) dans les trames 4,25-4,00 m et 4,00-3,75 $\mathrm{m}$ globalisées, sans indication de provenance de secteur.

Plus récemment, à l'occasion de sa thèse, J.E. AuraTortosa a procédé à un réexamen de la séquence postsolutréenne du Talud (couches 1 à 13). En complément à une analyse typologique des outillages lithiques et osseux, l'étude de la techno-morphologie des supports d'outils, de leur typométrie et des retouches latérales lui permet de proposer une nouvelle structuration des industries situées immédiatement après le SolutréoGravettien et de reconnaître, dans les couches 6 à 11, une nouvelle entité baptisée Magdalénien antique méditerranéen [MAM] de faciès Badegoulien type Parpalló, structuré en deux phases typologiques et chronologiques A (ancienne) et B (récente) (Aura-Tortosa, 1988, 1995 et 1997).

Enfin, dans un travail de synthèse sur les industries situées entre 21000 et 17500 BP en Europe sudoccidentale, nous avons démontré une forte régionalisation du Badegoulien, dont un faciès méditerranéen reconnu au Parpalló, à l'entrée de la grotte et dans le Talud, caractérisé par un équilibre typologique singulier (fort développement des grattoirs, sur lame retouchée ou de type aurignacien, des lames retouchées et des outils "archaïques", essentiellement des encoches et denticulés, rareté des burins, des perçoirs et des lamelles à dos) et structuré en deux phases chronologiques et industrielles (Bosselin, 2000).

La figure 2 résume les principaux modèles chronostratigraphiques et industriels de la séquence archéologique du Parpalló (Fortea, 1985; Fortea et Jordá, 1976; 


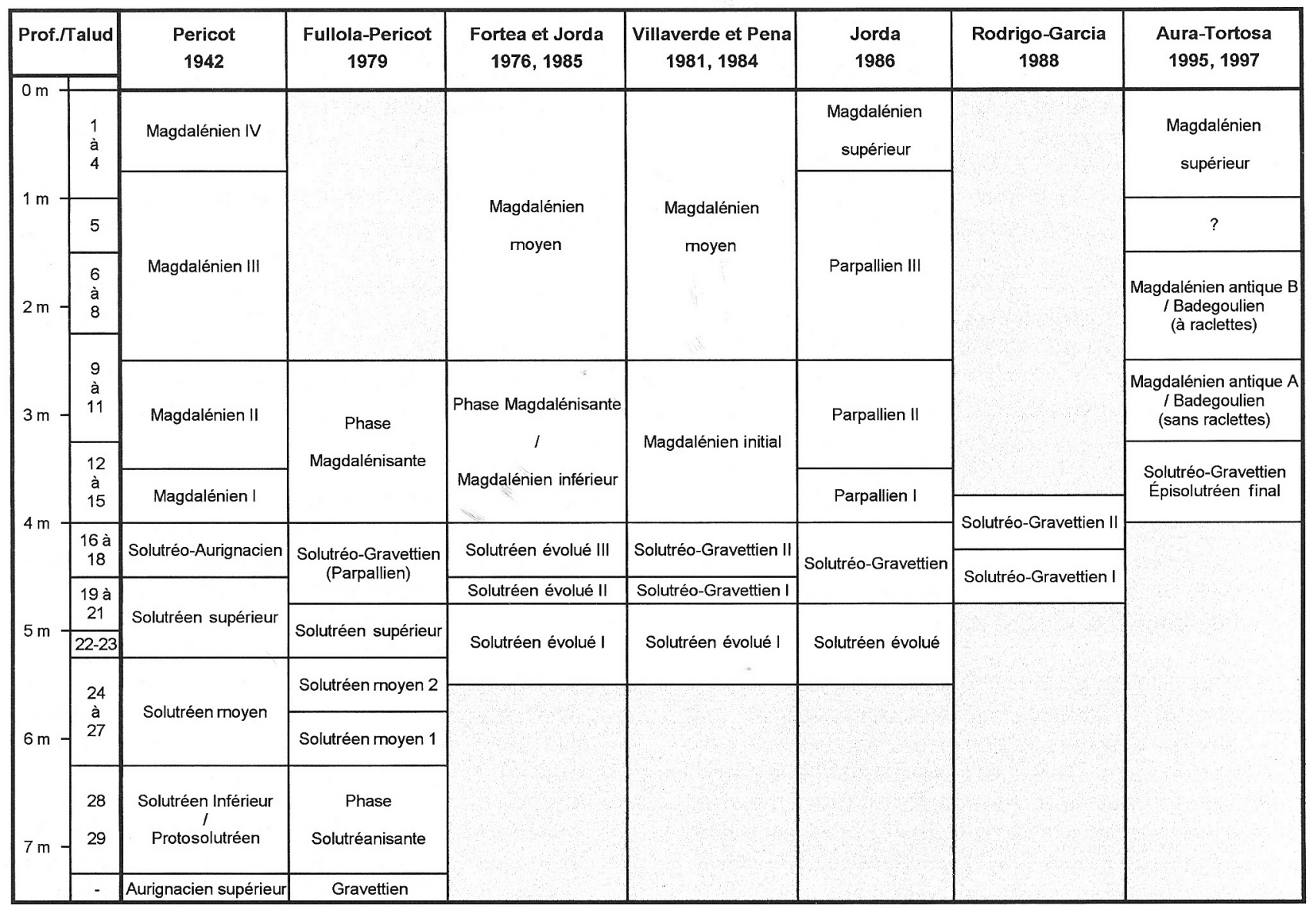

Fig. 2 - Le Parpalló. Principales propositions chronostratigraphiques historiques.

Fullola-Pericot, 1979; Jordá, 1986; Pericot, 1942; Rodrigo-Garcia, 1988; Villaverde, 1984; Villaverde et Peña, 1981).

Ces nouvelles données nous permettent aujourd'hui d'entreprendre une première étude quantitative de la morphométrie des produits de première intention (supports d'outils) issus du débitage de la séquence postsolutréenne du secteur du Talud, de proposer une structuration des industries lithiques et osseuses du Parpalló, de comparer les résultats obtenus entre ces deux approches et d'approfondir les modalités d'une éventuelle évolution entre le Badegoulien et le Magdalénien sur ce site.

\section{LE DÉBITAGE :
ÉTUDE TECHNO-MORPHOMÉTRIQUE}

\section{Les données et la méthodologie}

Les données publiées par J.E. Aura-Tortosa (AuraTortosa, 1995), sous la forme de tableaux d'effectifs ou de fréquence par couche, ne permettent pas de reconstituer les chaînes opératoires de débitage et leur évolution en stratigraphie. Ainsi, seule une étude globale des supports d'outils sera menée, sans prendre en compte la totalité des vestiges comme les "déchets" (assimilables à des produits d'intention dérivée, information in litteris de B. Valentin que nous remercions), dans le but de reconnaître et de décrire les possibles processus de production d'outils et leur variabilité dans la séquence. D'autres tableaux de données, divisés par grandes catégories d'outils (grattoirs, burins, perçoirs et becs...) n'ont pas été pris en compte dans notre analyse (AuraTortosa, 1995, p. 196-199). En effet, hormis les groupes typologiques des grattoirs, des encoches-denticulés et des lamelles à dos, qui constituent l'essentiel de l'outillage, les effectifs globaux sont trop limités pour assurer leur représentativité statistique, et valider les interprétations pouvant résulter de leur analyse.

Nous avons donc construit une typologie décrivant la techno-morphologie des supports d'outils, leur typométrie, dont les variables quantitatives ont été recodifiées de façon à obtenir un tableau homogène constitué de modalités ( $c f$. infra), et la caractérisation de la (des) retouche(s) latérale(s). Par rapport aux tableaux publiés par J.E. Aura-Tortosa (Aura-Tortosa, 1995, p. 192-193), les variables de techno-morphologie n'ont pas été recodifiées, hormis l'agrégation sous une même catégorie des talons dièdres et facettés. Par contre, des regroupements ont été effectués pour les données typométriques, afin d'éviter la construction, probable du fait de l'existence de modalités à faible effectif (principalement pour les classes "extrêmes" des variables longueur, indice d'allongement et indice d'épaississement ${ }^{2}$ ), d'axes factoriels parasites dont l'interprétation archéologique serait délicate, et surtout sujette à caution. 
Notre typologie se compose donc de 25 modalités correspondant à l'éclatement de 6 variables, complétées par une variable de présence/absence (cortex) :

\section{- Techno-Morphologie}

- Support : éclat, lame, lamelle, autre;

- Cortex : présence/absence;

- Talon : cortical, lisse, facetté + dièdre, punctiforme.

\section{- Typométrie}

- Longueur : très petit (moins de $20 \mathrm{~mm}$ ), petit (de 20 à $29 \mathrm{~mm}$ ), grand (de 30 à $39 \mathrm{~mm}$ ), très grand (plus de $40 \mathrm{~mm}$ )

- Allongement : très large $(\mathrm{L} / \mathrm{l}<1)$, large $(1<\mathrm{L} / 1<$ $1,5)$, long $(1,5<\mathrm{L} / \mathrm{l}<2,5)$, lame $(\mathrm{L} / \mathrm{l}>2,5)^{3}$;

Epaississement : très mince $(1 / \mathrm{e}>4)$, mince $(3<1 / \mathrm{e}$ $<4)$, épais $(2<1 / \mathrm{e}<3)$, très épais $(1 / \mathrm{e}<2)$.

\section{- Retouche latérale}

- Type de retouche : abrupte, simple, coup de burin, esquillée, surélevée + plane.

Le corpus de niveaux industriels pris en compte ici est constitué par les couches 1 à 13 du Talud publiées par J.E. Aura-Tortosa. Leur représentativité, qui a été parfois mise en cause du fait de l'ancienneté et des techniques de fouilles employées, a été confirmée par un pendage des couches en accord avec la documentation photographique disponible et par l'absence de sélection et/ou de tri du matériel (Aura-Tortosa, 1995, p. 41).

Le tableau de fréquence des modalités de variable ainsi constitué, sous sa forme canonique, est ensuite traité par des méthodes d'analyse des données : analyse factorielle des correspondances, puis classification ascendante hiérarchique (méthode des voisins réductibles) sur les axes factoriels dont l'inertie dépasse $5 \%$ de l'information initiale.

\section{Les résultats du traitement statistique}

Le premier axe de l'analyse factorielle des correspondances (52,8\% d'inertie) oppose d'une part les "supports éclats", à indice d'allongement très réduit (L/1 $<1$ : pièces très larges) et d'autre part les supports laminaires et lamellaires, les talons punctiformes, les pièces à indice d'allongement élevé (L/l > 1,5 : pièces laminaires et lames) et/ou la retouche latérale abrupte. Il révèle un antagonisme entre d'une part un débitage axé vers la production d'éclats et d'autre part un débitage axé vers la production de lames et de lamelles, vraisemblablement suivant plusieurs schémas opératoires (cf. infra).

Le second axe factoriel (31,0\% d'inertie) oppose d'une part les supports lamellaires, les talons punctiformes et/ou les retouches latérales abruptes et simples et d'autre part les supports laminaires, les talons lisses et/ou les pièces de module grand ou très grand (longueur $>30 \mathrm{~mm}$ ). Il révèle un antagonisme entre d'une part un débitage axé vers la production de lames et d'autre part un débitage axé vers la production de lamelles, vraisemblablement suivant plusieurs schémas opératoires ( $c f$. infra).
Le troisième axe factoriel enfin (7,5\% d'inertie) oppose d'une part les supports lamellaires et/ou les pièces à indice d'épaississement très réduit $(1 / \mathrm{e}<2$ : supports très épais) et d'autre part les talons punctiformes et/ou les pièces à indice d'épaississement élevé ou très élevé (1/e $>3:$ supports minces ou très minces). Il permet d'entrevoir la possibilité de l'existence d'au moins deux chaînes opératoires de débitage d'éclats, en introduisant une distinction dans l'ensemble défini sur le premier axe factoriel, dont les produits de première intention (les supports d'outils) ne possèdent pas les mêmes caractéristiques morphométriques.

La projection des niveaux archéologiques et des modalités de variables dans les plans factoriels 1-2 et 1-3 est présentée sur la figure 3 .

La classification automatique, effectuée sur les trois premières coordonnées factorielles, met en évidence une structure de partition en trois classes correspondant à trois traditions différentes de production de supports : - couches 1 à 5 : production préférentielle de supports lamellaires, puis laminaires, à talons punctiformes, puis lisses et enfin facettés, présentant un module plutôt petit (moins de $30 \mathrm{~mm}$ ), un indice d'allongement élevé ( $\mathrm{L} / \mathrm{l}>2,5$ : lames) et un indice d'épaississement élevé ( $1 / \mathrm{e}>3:$ pièces minces).

- couches 6 à 11 : production préférentielle d'éclats, fréquemment corticaux, à talons lisses et corticaux, présentant un module petit ou très petit (moins de $30 \mathrm{~mm}$ ), un indice d'allongement réduit ou très réduit $(\mathrm{L} / \mathrm{l}<1,5$ : éclats larges et très larges) et un indice d'épaississement réduit $(1 / \mathrm{e}<3$ : éclats épais et très épais).

À un niveau d'explication plus fin, cet ensemble se structure en deux phases : les couches 6 à 8 d'une part, les couches 9 à 11 d'autre part ( $c f$. infra).

- couches 12 et 13 : Production mixte, de supports laminaires ou d'éclats, à talons presque essentiellement lisses, présentant un module grand ou très grand (plus de $30 \mathrm{~mm}$ ), un indice d'allongement élevé (L/1 > 1,5 : éclats laminaires et lames) et un indice d'épaississement élevé $(1 / \mathrm{e}>3$ : pièces minces et très minces).

\section{Discussion des résultats}

L'emploi de techniques statistiques multivariées sur un tableau de données décrivant les caractéristiques techno-morphologiques et typométriques des supports d'outils nous a permis de distinguer trois "traditions" de production, correspondant aux trois classes de la classification automatique, axées vers l'obtention préférentielle d'éclats, de lames ou de lamelles. Chaque ensemble se caractérise par un équilibre singulier au niveau de la fréquence relative des modalités de variables prises en compte dans cette analyse, traduisant la variabilité diachronique de la techno-morphométrie des produits de première intention dans la séquence archéologique, et donc probablement de l'ensemble des chaînes opératoires de débitage.

Sur un plan technique, deux modes de production s'opposent vraisemblablement, orientés vers la réalisation 


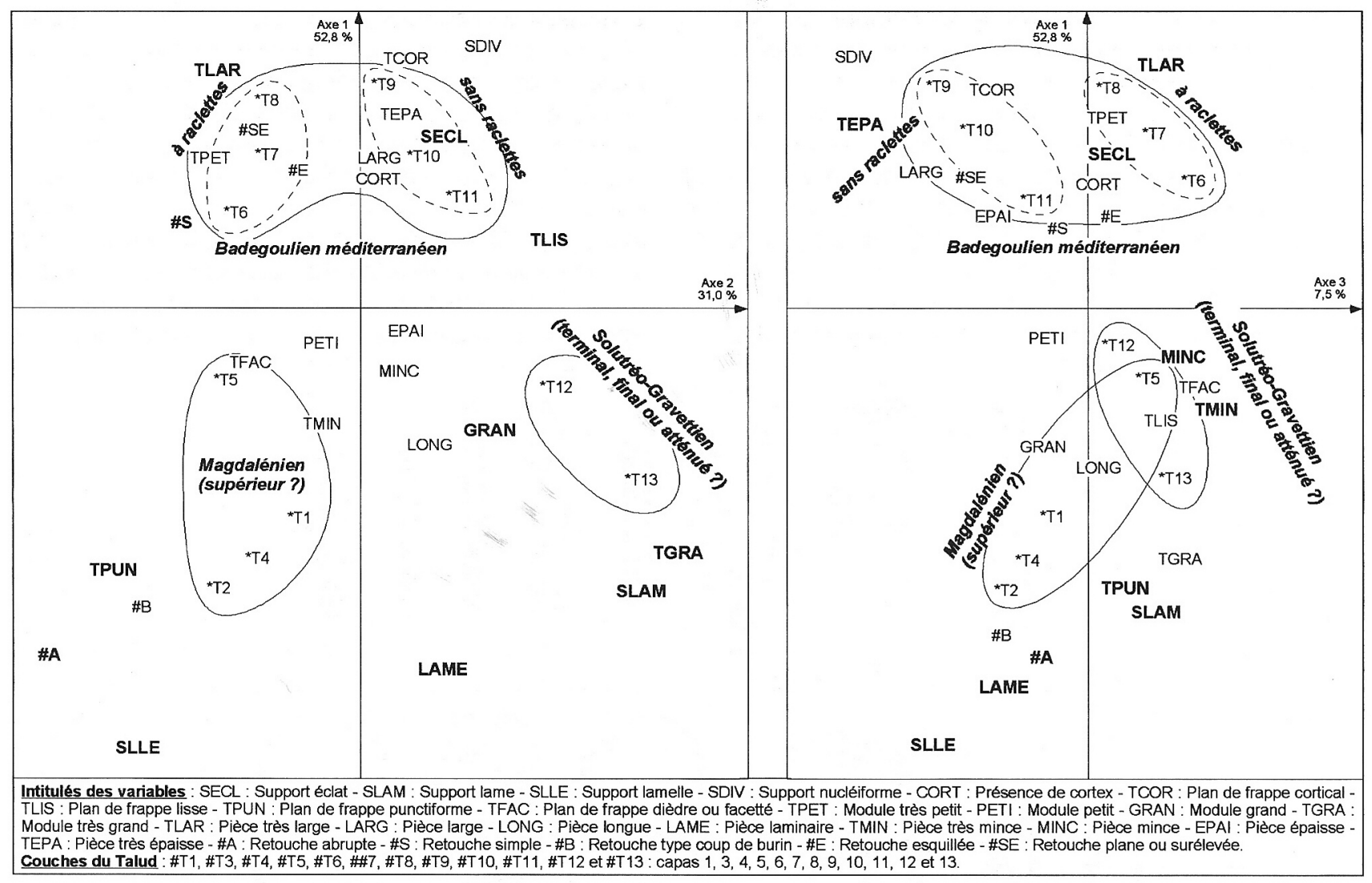

Fig. 3 - Le Parpalló-Talud. Analyse statistique de la morphométrie des supports d'outils : projection des variables et des couches dans les plans factoriels 1-2 et 1-3 (les variables responsables de la signification des axes sont figurées en gras).

de supports fins et élancés (lames et lamelles) pour le premier, et de pièces plus trapues et plus épaisses (éclats) pour le second. A l'intérieur du premier ensemble, la différenciation entre un débitage laminaire et un débitage lamellaire est visible sur le second axe factoriel. Sur les axes 2 et 3 , il est également possible de pressentir les indices de deux schémas opératoires de production d'éclats, conduisant à des supports à tendance d'une part large et très large, et d'autre part épaisse et très épaisse. Leurs caractéristiques typométriques nous permettent de les distinguer et de proposer une modélisation de leur évolution en stratigraphie. La production d'éclats conduit à des supports fréquemment corticaux, à talons lisses ou corticaux, possédant un module réduit, large et de forte épaisseur, s'inscrivant "grosso modo" dans un cercle. Le débitage "en tranches de saucisson", dont on sait maintenant qu'il est, hormis sur le site éponyme de Badegoule, rarement identifié dans le Badegoulien (Cazals, 2000; Cheynier, 1939; Cretin, 1996; Cretin et Le Licon-Julien, 1997; Fourloubey, 1996a et b; Morala, 1993), semble avoir été reconnu par J.E. Aura-Tortosa, qui propose une description précise des produits de première intention en résultant (Aura-Tortosa, 1995, p. 89). Comme d'autres méthodes de production d'éclats "normalisés", supports potentiels des raclettes, sont maintenant reconnues et décrites dans le sud-ouest de la France (Cretin, 1996; Cretin et Le Licon-Julien, 1997; Fourloubey, 1996b; Morala, 1993), on peut légitimement se poser la question quant à l'existence de cette chaîne opératoire dans la stratigraphie du Parpalló, en particulier pour la reconnaissance des éventuels éléments diagnostiques de ce type de débitage.

En l'absence d'une relecture de l'ensemble des vestiges disponibles (produits et déchets de débitage), il faut également être prudent quant à la reconnaissance et à la quantification des différents systèmes de production des lames et des lamelles. La mise en évidence, par des techniques factorielles, d'associations préférentielles dans les caractéristiques morphotechniques et métriques des supports d'outils engage notre réflexion quant à la possibilité de définir une méthodologie permettant de caractériser les chaînes opératoires de débitage présentes sur le site, leurs éléments technologiquement significatifs, les produits de première intention (les supports d'outils) et les déchets (produits d'intention dérivée) en résultant.

D'un point de vue diachronique, le Magdalénien des couches 1 à 5 se caractérise par un débitage axé vers la production de lamelles. La retouche abrupte domine les autres types, en rapport avec la fréquence des lamelles à dos dans ces ensembles. Le niveau de base (couche 5) s'en distingue sensiblement par une plus forte proportion d'éclats, ce qui pourrait indiquer soit une "phase intermédiaire" ou un mélange (lié aux techniques de fouilles employées ou à des phénomènes de perturbation post-dépositionnelle) avec le Badegoulien méditerranéen sous-jacent, soit un effet d'échantillonnage 
concentrant plus d'éclats dérivés des chaînes laminaires, comme les produits de mise en forme par exemple (Valentin, information in litteris).

Le Badegoulien méditerranéen révèle, en moyenne, une profonde cassure technologique et morphométrique avec l'ensemble sus-jacent, puisque le débitage est, à ce moment, orienté vers la production d'éclats. Il se structure en deux faciès techniques et chronologiques ancien et récent. Le Badegoulien méditerranéen récent (couches 6 à 8) se caractérise par des supports à talons lisses, corticaux et punctiformes, de morphologie large et relativement épaisse. Certains, à profil pseudoelliptique, peu allongés et relativement minces, confirment les indices d'un débitage orienté, suivant une ou plusieurs chaînes opératoires, vers l'obtention d'éclats de taille "normalisée", en liaison avec la présence significative des raclettes dans les outillages associés (Cretin, 1996; Cretin et Le Licon-Julien, 1997; Fourloubey, 1996b; Morala, 1993). Le Badegoulien méditerranéen ancien (couches 9 à 11) se caractérise par des supports à talons lisses ou corticaux, de module plus grand, moins trapus mais nettement plus épais que dans

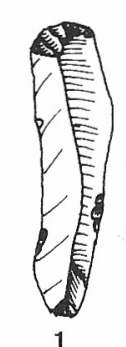

1

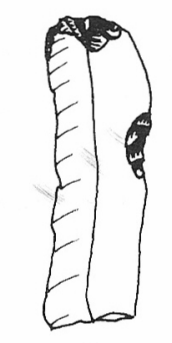

2
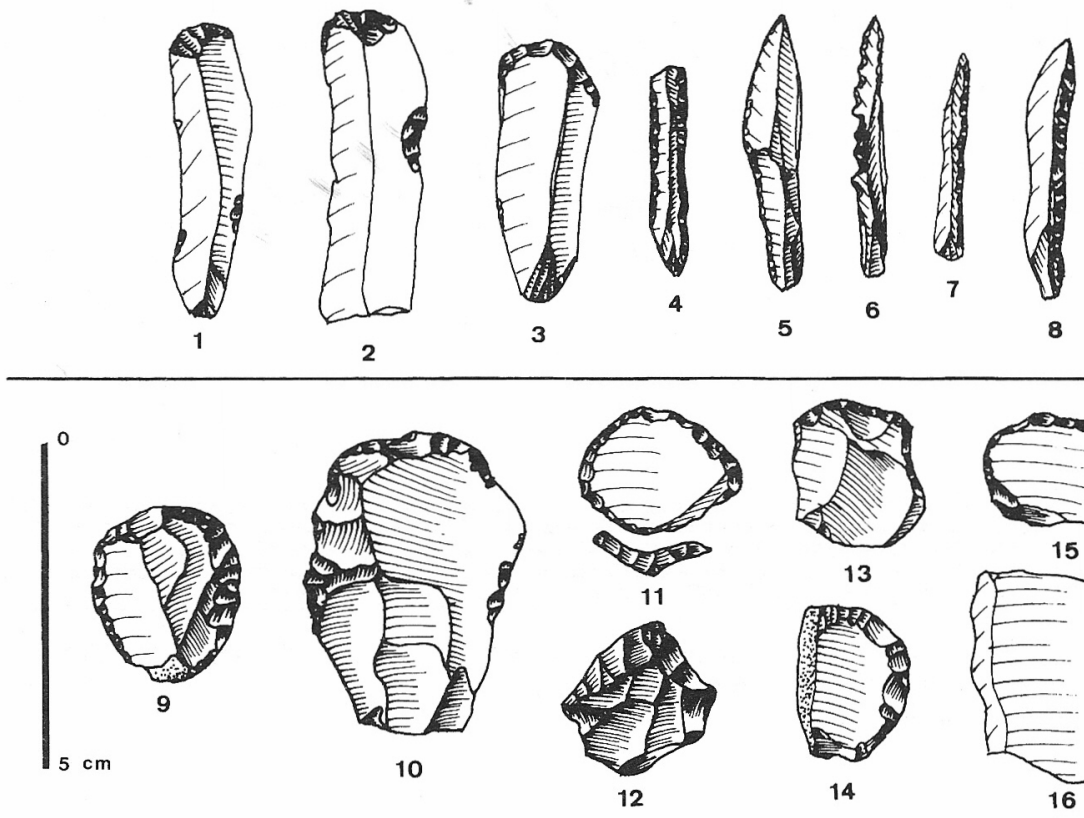

14
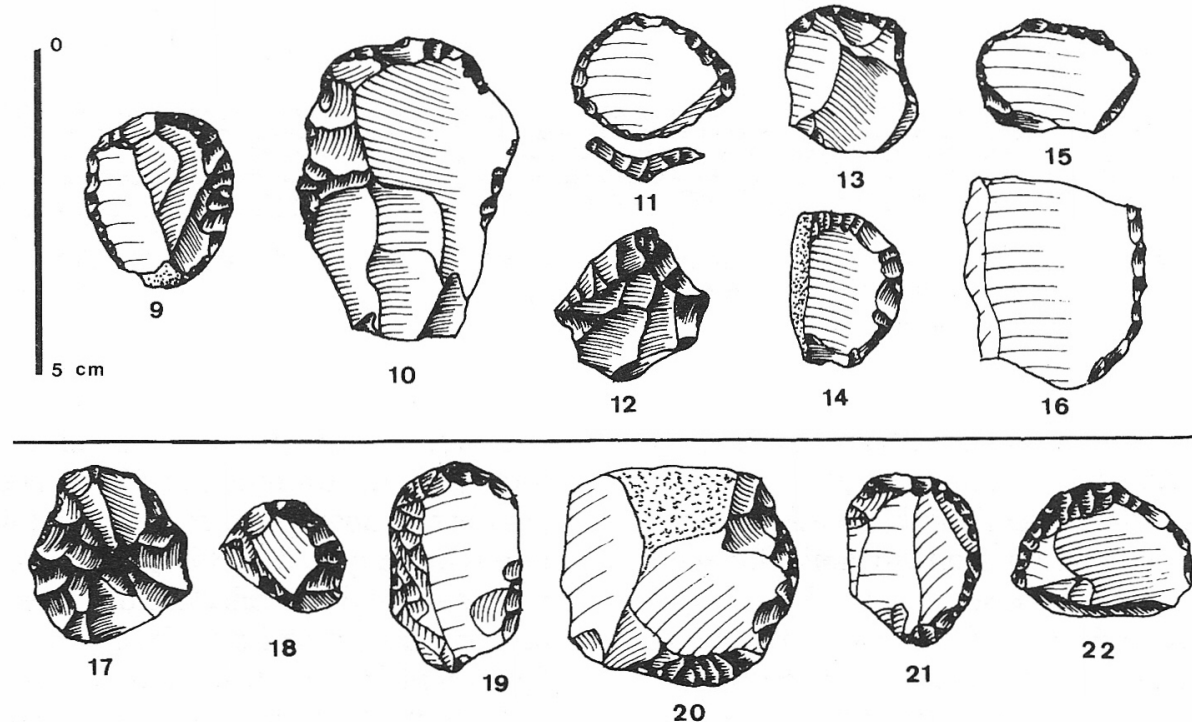

22

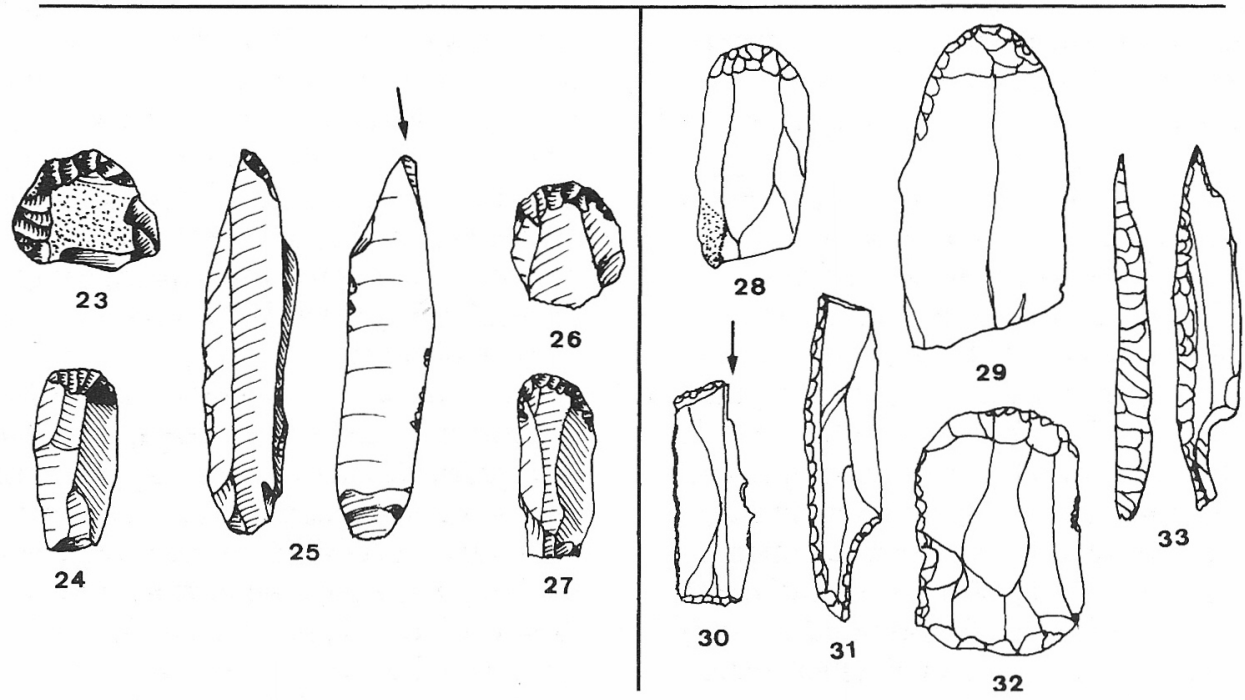

Fig. 4 - Le Parpalló-Talud. Industrie lithique. $\mathrm{n}^{\text {os }} 1-8$ : Magdalénien (couches 1 à 5) - $\mathrm{n}^{\text {os }}$ 9-16 : Badegoulien méditerranéen récent (couches 6 à 8) - $\mathrm{n}^{\text {os }}$ 17-22 : Badegoulien méditerranéen ancien (couches 9 à 11) - $\mathrm{n}^{\text {os }} 23-27$ : "inter Solutréo-Gravettien/Badegoulien" (couches 12 et 13) - nos 28-33 : Solutréo-Gravettien (couches 14 à 18). 
les ensembles sus-jacents. Ce changement dans la morphométrie des éclats-supports s'accompagne en outre de la quasi-disparition des raclettes.

Les couches 12 et 13, attribuées par J.E. Aura-Tortosa au Solutréo-Gravettien (Épisolutréen final), se caractérisent par la coexistence des trois types de production : d'éclats, de lames et, dans une moindre mesure, de lamelles. Malheureusement, l'impossibilité d'inclure dans cette étude les données sous-jacentes du SolutréoGravettien (couches 14 à 18) ne permet pas de préciser et/ou de confirmer les indices d'une éventuelle évolution technique (et typologique) graduelle du SolutréoGravettien vers le Badegoulien méditerranéen ${ }^{4}$.

La figure 4 propose une planche d'outillage traduisant les supports les plus typiques des différents modes de production décrits précédemment, respectivement pour le Magdalénien (couches 1 à 5), le Badegoulien méditerranéen récent (couches 6 à 8), le Badegoulien méditerranéen ancien (couches 9 à 11), "l'inter SolutréoGravettien/Badegoulien" (couches 12 et 13) d'après J.E. Aura-Tortosa (Aura-Tortosa, 1995), et pour le Solutréo-Gravettien (couches 14 à 18) d'après J.M. FullolaPericot (Fullola-Pericot, 1979).

Comme nous l'avions indiqué précédemment (Bosselin, 2000), si les modes de débitage du SolutréoGravettien, du Badegoulien méditerranéen et du Magdalénien montrent, en moyenne, des compositions différentes, l'existence d'une évolution progressive en stratigraphie constitue un élément important à prendre en compte pour mieux comprendre les rapports entre ces faciès archéologiques et régler définitivement la question de leur phylogénie.

\section{L'ANALYSE DES INDUSTRIES LITHIQUES ET OSSEUSES}

\section{L'analyse des industries lithiques}

Suivant une méthodologie désormais classique de l'analyse quantitative des industries lithiques paléolithiques (analyse factorielle des correspondances puis classification automatique), le traitement des données typologiques des couches 1 à 18 du Talud du Parpalló (Aura-Tortosa, 1995; Fullola-Pericot, 1979) conduit à une structuration en 6 classes $^{5}$ (fig. 5) :

- Couches 1 à 5 : elles se caractérisent par un outillage dominé par les lamelles à dos, la supériorité modérée des grattoirs sur les burins et la faible représentation des outils "archaïques" (encoches, denticulés, pièces esquillées, racloirs et raclettes) et des lames retouchées : Magdalénien (supérieur ?6).

- Couches 6 à 8 : elles se caractérisent par la fréquence des outils "archaïques", dont des raclettes, l'abondance des lames retouchées, des encoches et denticulés et la supériorité des grattoirs sur les burins : Badegoulien méditerranéen récent à raclettes (Magdalénien antique [de faciès Badegoulien] "type Parpalló" : phase B à raclettes de J.E. Aura-Tortosa). Dans cet ensemble, la couche 8 s'en distingue par un pourcentage plus réduit des raclettes, au profit des encoches-denticulés et des grattoirs de type aurignacien.
- Couches 9 à 11 : elles se caractérisent par l'abondance des outils "archaïques", essentiellement des encoches et denticulés, et des lames retouchées, la rareté ou 1'absence des raclettes et la forte supériorité des grattoirs sur les burins : Badegoulien méditerranéen ancien sans raclettes (Magdalénien antique [de faciès Badegoulien] "type Parpalló" : phase A sans raclettes de J.E. Aura-Tortosa).

- Couches 12 et 13 : elles se caractérisent par un équilibre typologique intermédiaire entre le Badegoulien méditerranéen ancien sans raclettes et le SolutréoGravettien, voyant la coexistence des pièces à cran et des outils "archaïques" et la fréquence des lames retouchées : Solutréo-Gravettien terminal; ou phase intermédiaire entre le Solutréo-Gravettien et le Badegoulien.

- Couches 14 à 16 : elles se caractérisent par la supériorité des grattoirs sur les burins, l'importance des lamelles à dos et, dans une moindre mesure, des pointes à cran de type méditerranéen (à retouche abrupte) : Solutréo-Gravettien 2.

- Couches 17 et 18 : elles se caractérisent également par la supériorité des grattoirs sur les burins, 1'importance des lamelles à dos et des pointes à cran de type méditerranéen (à retouche abrupte) : SolutréoGravettien 1.

- Le Solutréo-Gravettien 1 se distingue du SolutréoGravettien 2 par une plus forte représentation des pointes à cran de type méditerranéen, au détriment des grattoirs et des burins plus rares?

\section{L'analyse des industries osseuses}

L'industrie osseuse des couches 1 à 13 du Talud du Parpalló, caractérisée par une certaine monotonie, est principalement constituée de pointes de sagaies et de leurs fragments : pointes à base découpées, sagaies à biseau simple, long, large et concave, pointes doubles, les autres catégories de l'outillage ne représentant pas plus de $10 \%$.

Contrairement à ce qui se passe dans le sud-ouest de la France et dans les Cantabres, les pièces caractéristiques du Solutréen et du Badegoulien (bipointes de section circulaire à méplat central, sagaies à biseau en lancette, sagaies à fût décoré par motifs tectiformes suivant la technique de "pseudo-excision") sont absentes, bien que L. Pericot signale, dans la trame 4,00-3,50 m, un biseau en lancette décoré en chevrons, typique du Magdalénien I du Placard (Aura-Tortosa, 1995; Bosselin, 2000; Pericot, 1942).

Un double changement s'opère toutefois entre le Solutréo-Gravettien et le Badegoulien méditerranéen (entre les couches 15 et 12 du Talud), avec d'une part l'apparition du biseau simple à la fin du SolutréoGravettien II, au lieu de la base polygonale classique du Solutréen levantin, et d'autre part la substitution de l'os par le bois de cerf comme choix de support des sagaies (Aura-Tortosa, 1995, p. 105; Villaverde, 1992).

L'évolution de la fréquence relative des sections, présentée sur la figure 6, révèle 1'homogénéité de 1'ensemble "Solutréo-Gravettien terminal" (ou "inter- 


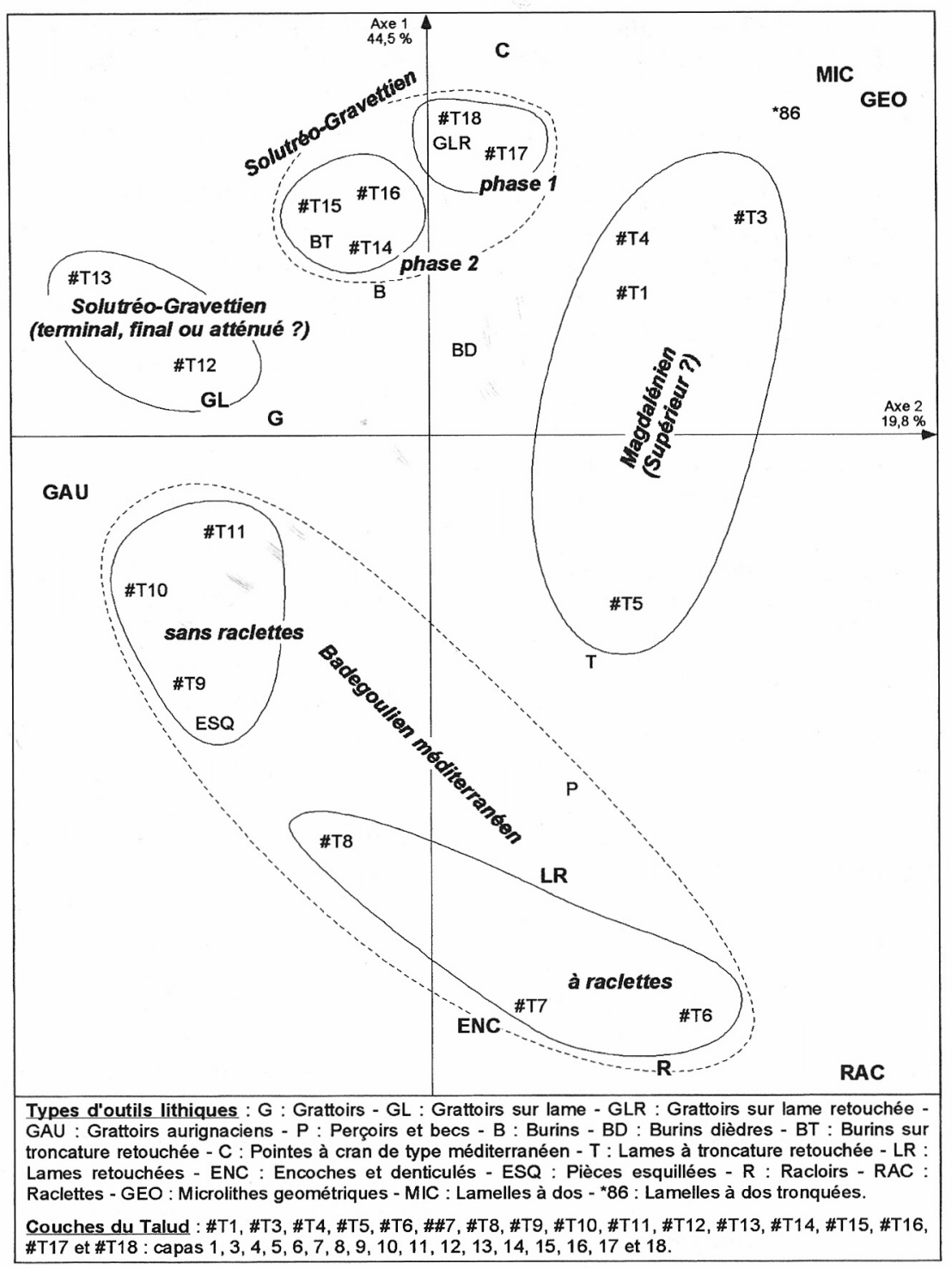

Fig. 5 - Le Parpalló-Talud. Analyse statistique des outillages lithiques : projection des types d'outils et des couches dans le plan factoriel 1-2 (les variables responsables de la signification des axes sont figurées en gras).

$\%$

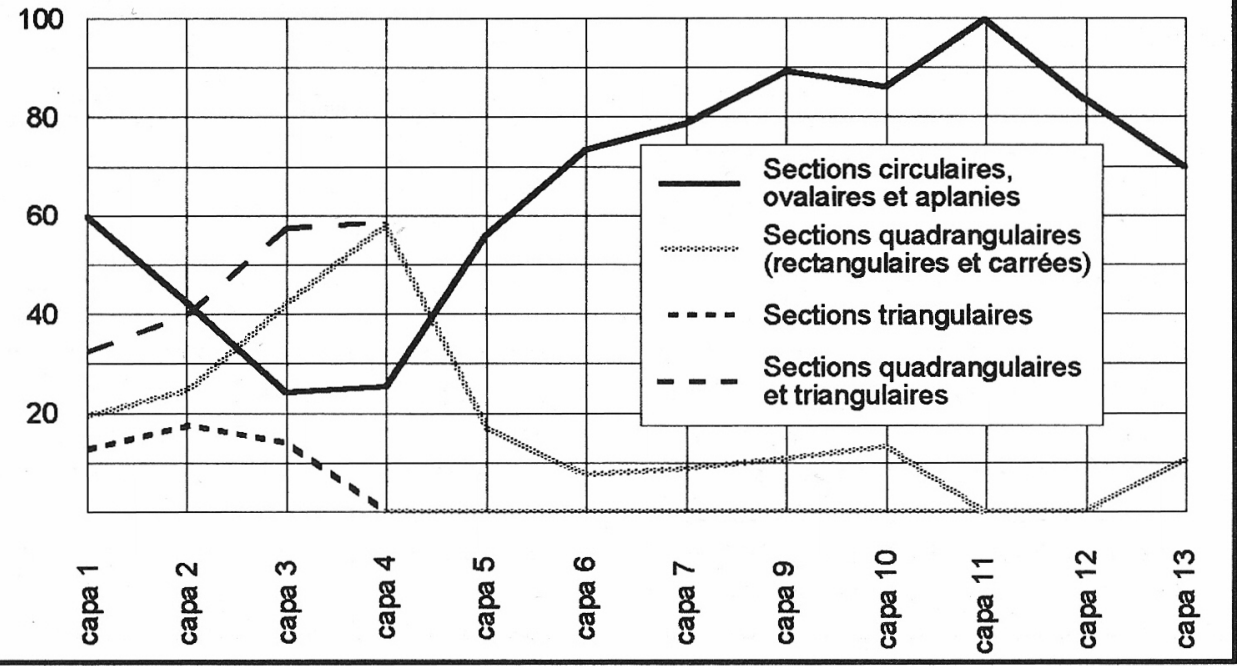

Fig. 6 - Industrie osseuse. Distribution des sections des sagaies dans la séquence du Talud. 
Solutréo-Gravettien/Badegoulien ")/Badegoulien méditerranéen des couches 6 à 13, dominé par les sections circulaires, ovalaires ou aplanies, avec une légère augmentation de ces dernières vers le haut de la séquence. Dès la base du Magdalénien (couche 5), on assiste à l'augmentation des sections quadrangulaires (carrées et rectangulaires), évolution qui s'amplifiera dans les niveaux plus récents (couches 1 à 4), comme dans les Cantabres d'ailleurs (Bosselin, 2000). Si les sections montrent une réelle différence entre le Badegoulien méditerranéen et le Magdalénien, les décors en angles (lignes croisées en " $\mathrm{x}$ "), les biseaux longs et les décorations ondulées, sinueuses ou en zigzag se retrouvent aussi bien dans les niveaux du Magdalénien (couches 1 à 5) que du Badegoulien méditerranéen (couches 6 à 9).

En conclusion, si les industries osseuses du SolutréoGravettien, du Badegoulien méditerranéen et du Magdalénien montrent, en moyenne, des compositions ou des caractéristiques stylistiques sensiblement différentes, quelques similitudes sont visibles et constituent des points de comparaison importants à prendre en compte pour mieux comprendre les rapports entre ces traditions.

\section{Discussion des résultats}

La confrontation des résultats des analyses menées ici sur la morphométrie des supports d'outils, l'équilibre statistique des industries lithiques et l'outillage osseux confirme la quasi-simultanéité des variations diachroniques rencontrées dans la stratigraphie du Talud du Parpalló, malgré quelques décalages résiduels.

Ainsi, il existe une forte corrélation entre les variations dans la fréquence des différents types de produits de première intention (supports d'outils), et leur morphométrie, issus des chaînes opératoires du silex et la composition des outillages lithiques : débitage axé vers la production de lames associé à une industrie dominée par les grattoirs longs, les burins et les lames retouchées ("inter Solutréo-Gravettien/Badegoulien" : couches 12 et 13), débitage axé vers la production d'éclats associé à une industrie dominée par les grattoirs, sur éclat ou aurignaciens, de mauvais burins, de courtes lames retouchées et des outils "archaïques" (Badegoulien méditerranéen : couches 6 à 11), et débitage axé vers la production de lames et de lamelles associé à une industrie dominée par les grattoirs longs, les burins dièdres et les lamelles à dos (Magdalénien : couches 1 à 5).

La composition interne de l'outillage osseux, en particulier les variations dans la section des sagaies, est également corrélée avec les caractéristiques typologiques des industries lithiques : abondance des sagaies de section circulaire, ovalaire ou aplanie dans les niveaux à rares lamelles à dos (couches 6 à 13 du Badegoulien méditerranéen et de "l'inter-Solutréo-Gravettien/ Badegoulien"); apparition puis développement des sagaies de section quadrangulaire (carrée ou rectangulaire) dans les ensembles à nombreuses lamelles à dos (couches 1 à 5 du Magdalénien), posant le problème des modalités du remplacement des pointes de projectile en matière organique par des pointes de projectile en matière minérale, comme dans le tardiglaciaire du Bassin parisien (Valentin, 1999). Complémentairement, une cassure apparaît entre le Solutréo-Gravettien et le Badegoulien méditerranéen, avec l'invention du biseau simple absent auparavant.

Ces données confirment que l'évolution des différentes composantes des systèmes techno-typologiques que

\begin{tabular}{|c|c|c|c|c|c|c|c|}
\hline \multicolumn{2}{|c|}{ Prof. I Talud } & L. Pericot & $\begin{array}{l}\text { J.M. Fullola- } \\
\text { Pericot }\end{array}$ & \multicolumn{2}{|c|}{$\begin{array}{l}\text { J.E. Aura-Tortosa } \\
\text { M.J. Rodrigo-Garcia }\end{array}$} & \multicolumn{2}{|r|}{$\begin{array}{l}\text { B. Bosselin } \\
\text { (cette étude) }\end{array}$} \\
\hline \multirow[t]{2}{*}{$0 \mathrm{~m}$} & \multirow{2}{*}{$\begin{array}{l}1 \\
\text { à } \\
4\end{array}$} & Magdalénien IV & \multirow{4}{*}{ Magdalénien } & $\begin{array}{c}1 \text { à } \\
3\end{array}$ & \multirow{2}{*}{$\begin{array}{l}\text { Magdalénien supérieur } \\
\text { (MSM-A / MSM-B) }\end{array}$} & \multirow{2}{*}{$\begin{array}{l}1 \\
\text { à } \\
4\end{array}$} & \multirow{2}{*}{$\begin{array}{l}\text { Magdalénien } \\
\text { (supérieur ?) }\end{array}$} \\
\hline & & \multirow[b]{3}{*}{ Magdalénien III } & & & & & \\
\hline \multirow[b]{2}{*}{$2 m-$} & 5 & & & 5 & $?$ & 5 & Transition / Mélange? \\
\hline & $\begin{array}{l}6 \\
\text { à } \\
8 \\
\end{array}$ & & & $\begin{array}{l}6 \\
\text { à } \\
8\end{array}$ & $\begin{array}{c}\text { Magdalénien antique } \\
\text { (MAM-B type Parpalló } \\
\text { à raclettes) }\end{array}$ & $\begin{array}{l}6 \\
\text { à } \\
8 \\
\end{array}$ & $\begin{array}{c}\text { Badegoulien } \\
\text { méditerranéen récent } \\
\text { (à raclettes) }\end{array}$ \\
\hline \multirow[t]{3}{*}{$3 m-1$} & $\begin{array}{c}9 \\
\text { à } \\
11\end{array}$ & \multirow[t]{2}{*}{ Magdalénien II } & \multirow{3}{*}{$\begin{array}{c}\text { " Phase } \\
\text { Magdalénisante " }\end{array}$} & $\begin{array}{c}9 \\
\text { à } \\
11\end{array}$ & $\begin{array}{c}\text { Magdalénien antique } \\
\text { (MAM-A type Parpalló } \\
\text { sans raclettes) }\end{array}$ & $\begin{array}{c}9 \\
\text { à } \\
11\end{array}$ & $\begin{array}{l}\text { Badegoulien } \\
\text { méditerranéen ancien } \\
\text { (sans raclettes) }\end{array}$ \\
\hline & \multirow{2}{*}{$\begin{array}{c}12 \\
\text { à } \\
15 \\
\end{array}$} & & & $\begin{array}{c}12 \\
\mathrm{a}\end{array}$ & Solutréo-Gravettien I & $\begin{array}{l}12 \\
13\end{array}$ & $\begin{array}{l}\text { Solutréo-Gravettien } \\
\text { terminal / Transition? }\end{array}$ \\
\hline & & Magdalénien I & & 14 & & \multirow{2}{*}{$\begin{array}{c}14 \text { à } \\
16\end{array}$} & \multirow{2}{*}{ Solutréo-Gravettien II } \\
\hline \multirow{2}{*}{$4 m-$} & 16 à & Solutréo-Aurianacien & \multirow{2}{*}{$\begin{array}{c}\text { Solutréo-Gravettien } \\
\text { (Parpallien) }\end{array}$} & \multirow{2}{*}{$\begin{array}{c}15 \\
15 \\
\dot{a} \\
18\end{array}$} & \multirow{2}{*}{$\begin{array}{l}\text { Solutréo-Gravettien II } \\
\text { Solutréo-Gravettien I }\end{array}$} & & \\
\hline & \begin{tabular}{|c|}
18 \\
19 à
\end{tabular} & \multirow[b]{2}{*}{ Solutréen supérieur } & & & & $\begin{array}{l}17 \\
18 \\
\end{array}$ & Solutréo-Gravettien I \\
\hline $5 \mathrm{~m}$ & \begin{tabular}{|c|}
21 \\
$22-23$ \\
\end{tabular} & & Solutréen supérieur & & & \multirow{3}{*}{$\begin{array}{c}19 ? \\
\text { à } \\
27 ?\end{array}$} & \multirow{3}{*}{ Solutréen } \\
\hline \multirow{3}{*}{$6 \mathrm{~m}-$} & 24 & \multirow{2}{*}{ Solutréen moyen } & Solutréen moyen 2 & & & & \\
\hline & 27 & & Solutréen moyen 1 & & & & \\
\hline & $\begin{array}{l}28 \\
\text { et } \\
29\end{array}$ & $\begin{array}{c}\text { Solutréen inférieur } \\
\text { Protosolutréen }\end{array}$ & $\begin{array}{c}\text { " Phase } \\
\text { Solutréanisante " }\end{array}$ & & & $28 ?$ & $\begin{array}{c}\text { Solutréen inférieur ou } \\
\text { Protosolutréen? }\end{array}$ \\
\hline
\end{tabular}

Fig. 7 - Les séquences de la Cova del Parpalló proposées par L. Pericot (1942), J.M. Fullola-Pericot (1979), M.J. Rodrigo-Garcia (1988) et J.E. Aura-Tortosa (1988, 1995, 1997) et B. Bosselin (cette étude). 
constituent les sociétés préhistoriques est généralement complexe et en étroite relation, même si quelques décalages chronologiques sont toujours visibles. La mise en évidence de corrélations, d'anti-corrélations et de phénomènes inertiels impose, avant tout travail de synthèse, la prise en compte de l'ensemble des vestiges archéologiques à notre disposition et la confrontation des résultats des diverses disciplines mises en œuvre pour leur étude.

A la lueur de ces résultats, nous proposons, sur la figure 7, une interprétation de la séquence industrielle du Parpalló. Peu de différences apparaissent entre les résultats de notre analyse et ceux proposés par J.E. Aura-Tortosa et M.J. Rodrigo-Garcia, hormis peut-être quelques divergences mineures quant à l'articulation des différentes phases du Solutréo-Gravettien (AuraTortosa, 1995 ; Rodrigo-Garcia, 1988).

\section{SYNTHESE - PERSPECTIVES}

Cette première approche quantitative concernant un sous-ensemble des témoins du débitage, par construction incomplète puisque limitée aux caractéristiques morphométriques des supports d'outils, confirme leur caractère structurant, au même titre que les données plus "classiques" des industries lithiques et osseuses. Si la reconstitution des chaînes opératoires de débitage n'a pu être entreprise, du fait même des données utilisées, la synchronisation des changements technotypologiques dans la stratigraphie du Parpalló, révélée par les variations dans la fréquence de leurs produits de première intention (les supports d'outils), leur morphométrie et leur évolution diachronique, nous a permis d'identifier les indices possibles de l'existence de plusieurs systèmes de production de supports, systèmes en étroite interrelation avec la composition et le style des outillages lithiques et osseux associés. Des tendances évolutives, concernant les dimensions et le volume des produits débités, ont été reconnues en stratigraphie, et leur influence sur la morphométrie et l'équilibre des industries mises en évidence.

Loin de vouloir opposer les approches technologiques et typologiques, qui au contraire mettent ici brillamment en évidence leur complémentarité, la prise en compte de l'ensemble des données disponibles, associant 1'étude de 1'approvisionnement en matières premières, la recherche, la caractérisation et la quantification des différentes chaînes opératoires de débitage, leur influence sur la composition et le style des industries lithiques et osseuses (et de l'art), permettra de mettre au point une méthodologie d'analyse pluridisciplinaire de l'ensemble des vestiges archéologiques dont les résultats, en particulier les synchronisations et/ou les éventuels décalages chronologiques, permettront un examen plus fin des groupes préhistoriques peuplant l'Europe sud-occidentale au cours du pléniglaciaire du Würm récent, entre environ $22000 \mathrm{BP}$ et $15000 \mathrm{BP}$, et dont l'articulation n'est pas encore connue avec certitude.

\section{Remerciements :}

Nous tenons à remercier ici J.E. Aura-Tortosa pour l'envoi de sa monographie du site du Parpalló, J.M. FullolaPericot pour les renseignements qu'il nous a fournis sur le Solutréo-Gravettien de ce site, J.-P. Fagnart pour sa relecture et ses critiques constructives, et B. Valentin pour ses remarques, toujours éclairées et toujours pertinentes, qui, dans son rôle de relecteur, ont permis d'améliorer ce manuscrit.

\section{NOTES}

(1) Tout comme l'étude de la provenance des matières premières lithiques d'ailleurs.

(2) Pour la longueur : agrégation des modalités 0-9 et 10-19 mm et des modalités 40-49, 50-59 et > $60 \mathrm{~mm}$. Pour 1'indice d'allongement : agrégation des modalités $0-0,5$ et $0,5-1$ et des modalités $2,5-4,4-6,5$ et $>6,5$. Pour l'indice d'épaississement : agrégation des modalités $>5$ et 5-4 et des modalités $2-1$ et $<1$.

(3) On pourrait objecter une redondance entre certaines données qualitatives (Éclat/Lame/Lamelle) et métriques (indice d'allongement L/1). On constate en effet une corrélation entre les supports "Lame" et "Lamelle" sur le premier axe factoriel, mais également leur opposition sur le deuxième axe, ce qui confirme que ces deux variables ne sont pas strictement corrélées, et ne remet pas en cause la validité des résultats de l'analyse statistique présentée ci-après.

(4) Toutefois, les planches de dessins de l'outillage du SolutréoGravettien des couches 14 à 18 (Fullola-Pericot, 1979) indiquent clairement le choix de lames allongées et minces, et plus accessoirement de lamelles, comme supports d'outils pour ces niveaux ( $c f$. fig. 4).

(5) Suivant une même méthodologie, le traitement statistique de l'ensemble des niveaux de l'entrée de la grotte et du Talud conduit à une structuration en 7 classes : Solutréen (trames 4,75 à $7,25 \mathrm{~m}$ ), dont le Solutréen supérieur à feuilles de laurier à base concave et pointes à pédoncule et ailerons (trames 4,75 à 5,25 m), Solutréo-Gravettien 1 (trames 4,25 à 4,75 m - Talud couches 17 et 18), Solutréo-Gravettien 2 (trames 3,75 à $4,25 \mathrm{~m}$ - Talud couches 14 à 16), "inter SolutréoGravettien/Badegoulien" (trames 3,25 à 3,8 m - Talud couches 12 et 13), Badegoulien ancien sans raclettes (trames 2,00 à 3,25 m - Talud couches 9 à 11), Badegoulien récent à raclettes (trames 1,50 à 2,00 $\mathrm{m}$ - Talud couches 6 à 8) et Magdalénien (trames 0,00 à 1,60 m - Talud couches 1 à 5). De façon à ne pas alourdir la discussion, ces résultats ne seront pas développés plus précisément.

(6) Une seule datation absolue a été produite pour le "Magdalénien III" : (Birm-519) $13800 \pm 380$ BP (trame 1,75-1,50 m).

(7) En accord avec la division généralement admise du SolutréoGravettien : Solutréo-Gravettien I typique à nombreux crans et SolutréoGravettien II atténué à plus rares crans (Fortea, 1985; Fortea et Jordá, 1976; Rodrigo-Garcia, 1988; Villaverde, 1984; Villaverde et Peña, 1981).

\section{RÉFÉRENCES BIBLIOGRAPHIQUES}

AURA-TORTOSA J.E. (1988) - La Cova del Parpalló y el Magdaleniense de facies ibérica o méditeráneo. Propuesta de sistematización de su cultura material : industria lítica y ósea, Thèse, Universitat de València.

AURA-TORTOSA J.E. (1995) - El Magdaleniense Mediterráneo : La Cova del Parpalló (Gandia, Valencia), Diputación Provincial de Valencia, Servicio de Investigación Prehistórica, Serie de Trabajos Varios, 91, Valencia.
AURA-TORTOSA J.E. (1997) - Al sur del Ebro. Badeguliense y Magdaleniense en la región mediterránea (ca. 17000-11000 BP), in J. FULLOLA i N. SOLER dir., El món mediterrani després del pleniglacial (18000-12000 BP), Museu d'Arqueologia de Catalunya, Centre d'Investigacions Arqueològiques, Monogràfica 17, Girona, p. 243-253.

BOSSELIN B. (2000) - Le Badegoulien en Europe sud-occidentale : faciès régionaux, paléo-environnements et filiations, in : $3^{e}$ Congresso 
de Arqueologia Peninsular, Vila-Real, Septembre 1999, Éd. ADECAP, Porto, vol. 2 : Paleolítico da Península Ibérica, p. 363-401.

CAZALS N. (2000) - Constantes et variations des traits techniques et économiques entre le Magdalénien inférieur et moyen : analyse des productions lithiques du Nord de la péninsule ibérique, Thèse, Université de Paris I.

CHEYNIER A. (1939) - Le Magdalénien primitif de Badegoule. Niveaux à raclettes, Bulletin de la Société préhistorique française, $\mathrm{t}$. 36 , p. $354-396$

CRETIN C. (1996) - Vers une nouvelle perception du Badegoulien des Jamblancs. Premiers éléments techno-économiques, Paléo, 8, p. 243-268.

CRETIN C., LE LICON-JULIEN G. (1997) - Premières comparaisons sur la technologie du débitage du Magdalénien ancien : les Jamblancs (Dordogne, France) et l'abri Fritsch (Indre, France), Paléo, 9, p. 245-262.

FORTEA J. (1985) - El Paleolítico y Epipaleolítico en la Regíon Central del Mediterráneo peninsular. Estado de la cuestión industrial, in : Arqueología del País Valenciano: Panorama y perspectivas, Alicante, p. 31-52.

FORTEA J., JORDA F. (1976) - La Cueva de les Mallaetes y los problemas del Paleolítico Superior del Mediterráneo español, Zephyrus, 36-37, p. 129-166.

FOURLOUBEY C. (1996a) - Étude de la variabilité des industries de transition vers le Magdalénien par l'analyse des travaux de la taille du silex. L'exemple du Badegoulien et du Magdalénien ancien de plein air de la moyenne vallée de l'Isle (Dordogne), Thèse de $3^{\mathrm{e}}$ cycle, Université de Bordeaux I, 190 p.

FOURLOUBEY C. (1996b) - La production de raclettes au Chatenet (Saint-Front-de-Pradoux, Dordogne), Paléo, 8, p. 269-275.

FULLOLA-PERICOT J.M. (1979) - Las industrias líticas del Paleolitico superior Iberico. Diputación Provincial de Valencia, Servicio de Investigación Prehistórica, Serie de Trabajos Varios, 60, Valencia.

JORDA F. (1986) - Paleolítico, in : Prehistoria de España, Ed. Gredos, Madrid.
MORALA A. (1993) - Technologie lithique du Magdalénien ancien de l'abri Casserole (Les Eyzies-de-Tayac, Dordogne). Résultats préliminaires: schémas de production des supports pour les niveaux 6, 5 et 4, Paléo, 5, p. 193-208

PERICOT L. (1942) - La Cova del Parpalló (Gandía, Valencia), Publicaciones CSIC, Instituto Diego Velásquez, Madrid.

RODRIGO-GARCIA M.J. (1988) - El Solútreo-gravetiense de la Cova del Parpalló (Gandía) : algunas consideraciones sobre el solútreogravetiense en la secuencia del Paleolítico superior del área mediterránea peninsular, Saguntum, 21, p. 9-46

SONNEVILLE-BORDES D. de, PERROT J. (1954-1956) - Lexique typologique du Paléolithique supérieur, Bulletin de la Société préhistorique française, t. 51 (7), 1954, p. 327-335; t. 52 (2), 1955, p. 76-79; t. 53 (8), 1956, p. 408-412, et t. 53 (9), 1956, p. 547-549.

VALENTIN B. (1999) - Techniques et cultures : les chasseurs-cueilleurs de la fin du Tardiglaciaire au sud du Bassin parisien, in P. BINTZ et A. THEVENIN dir., L'Europe des derniers chasseurs - Épipaléolithique et Mésolithique, Ve Congrès UISPP, Grenoble, Septembre 1995, CTHS, Paris, p. 201-212.

VILLAVERDE V. (1984) - La industria magdaleniense de l'Abric de la Senda Vedada (Sumarcàrcer, Valencia). Nuevas consideraciones sobre el Magdaleniense Mediterráneo peninsular, Saguntum, 18, p. 29-47.

VILLAVERDE V. (1992) - El Paleolítico en el País Valenciano, Actas del Congreso "Aragón/Litoral Mediterráneo: intercambios culturales durante la Prehistoria”, p. 55-87, Zaragoza.

VILLAVERDE V., PEÑA J.L. (1981) - Piezas con escotadura del Paleolítico Superior valenciano, Diputación Provincial de Valencia, Servicio de Investigación Prehistórica, Serie de Trabajos Varios, 69, Valencia.

Bruno BOSSELIN

134, rue Gambetta, F-92150 Suresnes bosselin@worldnet.fr 